\title{
Hypercohones D-G, New Polycyclic Polyprenylated Acylphloroglucinol Type Natural Products from Hypericum cohaerens
}

\author{
Jing-Jing Zhang $\cdot$ Xing-Wei Yang $\cdot$ Jun-Zeng Ma $\cdot$ \\ Xia Liu $\cdot$ Li-Xin Yang $\cdot$ Sheng-Chao Yang $\cdot$ \\ Gang Xu
}

Received: 13 January 2014/ Accepted: 24 February 2014/Published online: 18 March 2014

(C) The Author(s) 2014. This article is published with open access at Springerlink.com

\begin{abstract}
Four new polycyclic polyprenylated acylphloroglucinol type metabolites, hypercohones D-G (1-4), along with four known analogues (5-8), were isolated from the aerial parts of Hypericum cohaerens. The structures of these isolates were elucidated by extensive spectroscopic methods. The inhibitory activities of these isolates against five human cancer cell lines in vitro were also tested.
\end{abstract}

Keywords Guttiferae $\cdot$ Hypericum cohaerens $\cdot$ Acylphloroglucinol $\cdot$ Hypercohones D-G

\section{Introduction}

Polycyclic polyprenylated acylphloroglucinols (PPAPs), with a highly oxygenated and densely substituted bicyclo[3.3.1]nonane-2,4,9-trione or other related core structures decorated with prenyl or geranyl side chains, are a kind of natural products from Hypericum, Clusia, and Garcinia plants in family Guttiferae [1-3]. This kind of

Electronic supplementary material The online version of this article (doi:10.1007/s13659-014-0007-5) contains supplementary material, which is available to authorized users.

J.-J. Zhang $\cdot$ X.-W. Yang · J.-Z. Ma $\cdot$ X. Liu $\cdot$ L.-X. Yang G. Xu ( $\square)$

State Key Laboratory of Phytochemistry and Plant Resources in West China, Kunming Institute of Botany, Chinese Academy of Sciences, Kunming 650201, People's Republic of China e-mail: xugang008@mail.kib.ac.cn

J.-J. Zhang $\cdot$ X.-W. Yang

University of Chinese Academy of Sciences, Beijing 100049,

People's Republic of China

S.-C. Yang $(\bowtie)$

Institute of Chinese Medicinal Materials, Yunnan Agricultural University, Kunming 650201, People's Republic of China

e-mail: shengchaoyang@163.com metabolites showed a wide variety of biological activities such as antitumor, antimicrobial, anti-HIV, antioxidant, and especially in the central nervous system as modulators of neurotransmitters associated with neuronal damage and depression [4-9]. Hyperforin was the most famous one in acylphloroglucinol family isolated from $H$. perforatum (St. John's Wort) [11], and was reported to possess significant antidepressant, anticancer, and antibiotic activities [10].

The plants of genus Hypericum, occurring widely in temperate regions, have been used as traditional medicines in many countries all over the world [12]. H. cohaerens $\mathrm{N}$. Robson was an endemic plant distributing in Guizhou and Yunnan provinces, P. R. China [13]. A polyprenylated acylphloroglucinol derivative with a novel bicyclo[5.3.1]hendecane core (hypercohin A) and twelve other PPAP type natural products have been reported from this plant previously [14-16]. In our systematic search for new and bioactive PPAPs, a phytochemical investigation on the aerial parts of $H$. cohaerens was carried out and four new PPAP type metabolites (hypercohones $D-G, 1-4)$ were isolated along with four known metabolites 5-8. It was notable that $\mathbf{4}$ was a spiro-cyclic type PPAP, and also was the fifth natural product with such a complex ring system. Reported herein, was the isolation, structural elucidation, and the cytotoxic bioassay of these PPAP type metabolites. 


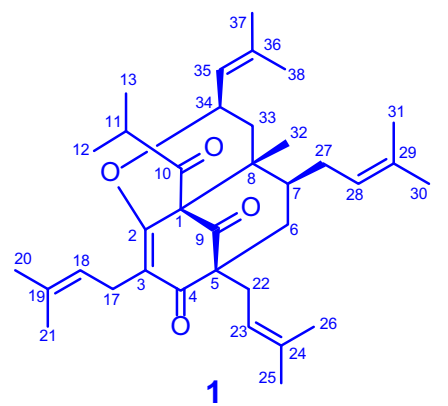<smiles>CC(C)=CCC[C@@]1(C)C(=O)C2(C(=O)c3ccccc3)C(=O)C(CC=C(C)C)=C3OC(C(C)(C)O)C[C@]32C[C@@H]1CC=C(C)C</smiles>

\section{Results and Discussion}

The $\mathrm{MeOH}$ extract of the air-dried and powdered aerial parts of $H$. cohaerens $(10.0 \mathrm{~kg})$ was subjected to a silica gel column to afford five fractions A-E. Fraction B was subjected to a series of chromatographic methods, and led to the isolation of four new acylphloroglucinol derivatives, hypercohones D-G (1-4), together with four known ones, uralodin A (5), [17] uralodin B (6), [18] oxepahyperforin (7) [19], and tomoeone E (8) [20].

Compound 1 was obtained as a light yellow gum. Its molecular formula $\mathrm{C}_{35} \mathrm{H}_{50} \mathrm{O}_{4}$ was deduced on the basis of its positive HRESIMS peak at $\mathrm{m} / z, 557.3606[\mathrm{M}+\mathrm{Na}]^{+}$ (calcd for 557.3606), indicating 11 indices of hydrogen deficiency. The IR spectrum showed 1731 and $1665 \mathrm{~cm}^{-1}$ due to carbonyl functionalities. The ${ }^{13} \mathrm{C}$ NMR and DEPT spectra of $\mathbf{1}$ exhibited signals for 35 carbons, including twelve quaternary carbons (including three carbonyl carbons, five olefinic ones, and one oxygenated $\mathrm{sp}^{2}$ carbon), seven methines (including four unsaturated ones and an oxygenated one), five methylenes, and eleven methyls. These data indicated the characteristic signals for an acylphloroglucinol core with two nonconjugated carbonyl groups $\left(\delta_{\mathrm{C}} 208.3 \mathrm{C}-9 ; \delta_{\mathrm{C}} 210.7, \mathrm{C}-10\right)$, an enolized 1,3diketo group $\left(\delta_{\mathrm{C}} 170.4, \mathrm{C}-2 ; \delta_{\mathrm{C}} 133.9, \mathrm{C}-3 ; \delta_{\mathrm{C}} 196.8, \mathrm{C}-4\right)$, and two quaternary carbons at $\delta_{\mathrm{C}} 73.9(\mathrm{C}-1)$, and $\delta_{\mathrm{C}} 66.9$ (C-5) [17-19] The ${ }^{1} \mathrm{H}$ NMR spectrum of $\mathbf{1}$ showed the presence of four isoprenyl groups $\left[\delta_{\mathrm{H}} 5.08(1 \mathrm{H}, \mathrm{m}, \mathrm{H}-18)\right.$, $\delta_{\mathrm{H}} 5.06(1 \mathrm{H}, \mathrm{m}, \mathrm{H}-23), \delta_{\mathrm{H}} 5.05(1 \mathrm{H}, \mathrm{m}, \mathrm{H}-28), \delta_{\mathrm{H}} 5.18$ $(1 \mathrm{H}, \mathrm{d}, J=8.3 \mathrm{~Hz}, \mathrm{H}-35)]$ and an isopropyl group $\left[\delta_{\mathrm{H}}\right.$ $2.38(1 \mathrm{H}, \mathrm{m}, \mathrm{H}-11), \delta_{\mathrm{H}} 0.97(3 \mathrm{H}, \mathrm{d}, J=6.6 \mathrm{~Hz}, \mathrm{H}-12), \delta_{\mathrm{H}}$

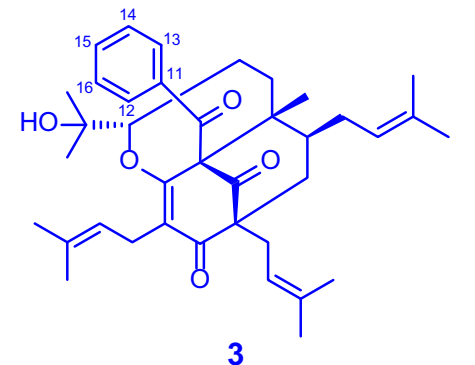

3

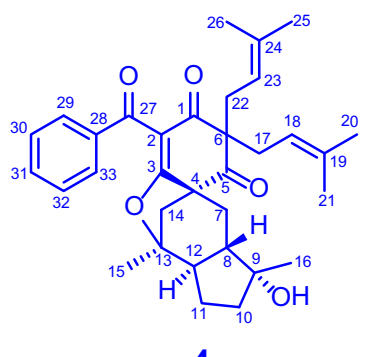

4

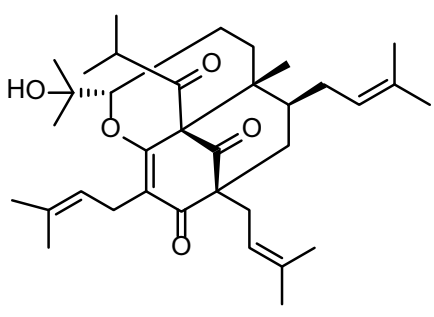

7

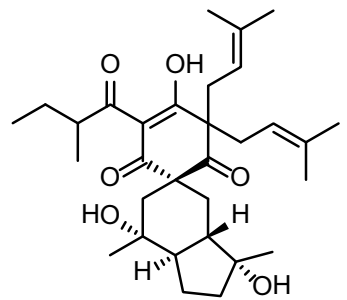

8
$1.03(3 \mathrm{H}, \mathrm{d}, J=6.6 \mathrm{~Hz}, \mathrm{H}-13)]$ (Table 2). Based on these data, 1 was considered as an acylphloroglucinol derivative having four isoprenyl groups and an isopropyl group. The HMBC correlations from $\mathrm{H}-17$ at $\delta_{\mathrm{H}} 3.15(1 \mathrm{H}$, dd, $J=13.8,8.4 \mathrm{~Hz}, \mathrm{H}-17 \mathrm{a}), \delta_{\mathrm{H}} 3.08(1 \mathrm{H}, \mathrm{dd}, J=13.8$, $6.6 \mathrm{~Hz}, \mathrm{H}-17 \mathrm{~b})$ to $\mathrm{C}-2\left(\delta_{\mathrm{C}} 170.4\right), \mathrm{C}-3\left(\delta_{\mathrm{C}} 133.9\right)$, and $\mathrm{C}-4$ $\left(\delta_{\mathrm{C}} 196.8\right)$, from $\mathrm{H}-22\left(\delta_{\mathrm{H}} 2.41,2 \mathrm{H}, \mathrm{m}\right)$ to $\mathrm{C}-4\left(\delta_{\mathrm{C}} 196.8\right)$, C-5 $\left(\delta_{\mathrm{C}} 66.9\right), \mathrm{C}-6\left(\delta_{\mathrm{C}} 41.71\right)$, and C-9 $\left(\delta_{\mathrm{C}} 208.3\right)$, and the spin-spin system of $\mathrm{H}_{2}-6 / \mathrm{H}-7 / \mathrm{H}-27 / \mathrm{H}-28$ obtained from the ${ }^{1} \mathrm{H}-{ }^{1} \mathrm{H}$ COSY spectrum suggested the three isoprenyls were located at C-3, C-5, and C-7, respectively. Then, the remained isobutyryl was deduced to locate at $\mathrm{C}-1$.

Further analysis of the NMR data of $\mathbf{1}$ with those of hyperforin revealed that they were structurally similar to each other except that the signals for the methylene at C-34 in hyperforin was replaced by an oxygenated methine $\left(\delta_{\mathrm{C}}\right.$ 80.9) in 1, [10, 11] as evidenced by the $\mathrm{H}_{2}-33 / \mathrm{H}-34 / \mathrm{H}-35$ unit observed in the ${ }^{1} \mathrm{H}-{ }^{1} \mathrm{H}$ COSY spectrum (Fig. 1). The existence of the epoxy group between $\mathrm{C}-34$ and $\mathrm{C}-2$ $\left(\delta_{\mathrm{C}} 170.4\right)$ can be fully confirmed by the 11 indices of hydrogen deficiency. In addition, the HMBC correlations from Me-32 $\left(\delta_{\mathrm{H}} 1.08,3 \mathrm{H}, \mathrm{s}\right)$ to $\mathrm{C}-1\left(\delta_{\mathrm{C}} 73.9\right), \mathrm{C}-7\left(\delta_{\mathrm{C}}\right.$ $39.5), \mathrm{C}-8\left(\delta_{\mathrm{C}} 49.0\right)$, and C-33 ( $\left.\delta_{\mathrm{C}} 37.3\right)$ confirmed the structures furthermore.

The relative configuration of compound $\mathbf{1}$ was determined on the basis of a ROESY experiment (Fig. 1). The ROESY correlations of Me-32/H-27, Me-32/H-33 $\beta, \mathrm{H}-34 /$ $\mathrm{H}-33 \alpha, \mathrm{H}-34 / \mathrm{H}-7$, and Me-12/H-35, indicated that $\mathrm{H}-7$ and $\mathrm{H}-34$ were both $\alpha$-oriented and Me-32 was $\beta$-oriented. Furthermore, the relative configurations of C-1 and C-5 in 1 were elucidated to be the same with those of hyperforin, 
Table $1{ }^{13} \mathrm{C}$ NMR data for compounds $\mathbf{1}-\mathbf{3}$ in $\mathrm{CD}_{3} \mathrm{OD}$ ( $\delta$ in ppm)

\begin{tabular}{|c|c|c|c|}
\hline Position & $1^{\mathrm{a}}$ & $2^{a}$ & $\mathbf{3}^{\mathrm{b}}$ \\
\hline 1 & 73.9, C & $77.8, \mathrm{C}$ & $73.3, \mathrm{C}$ \\
\hline 2 & $170.4, \mathrm{C}$ & 167.1, C & 169.0, C \\
\hline 3 & $133.9, \mathrm{C}$ & $134.4, \mathrm{C}$ & $128.5, \mathrm{C}$ \\
\hline 4 & $196.8, \mathrm{C}$ & 199.0, C & 196.0, C \\
\hline 5 & $66.9, \mathrm{C}$ & $66.8, \mathrm{C}$ & $65.9, \mathrm{C}$ \\
\hline 6 & $41.71, \mathrm{CH}_{2}$ & $43.2, \mathrm{CH}_{2}$ & 41.6, $\mathrm{CH}_{2}$ \\
\hline 7 & $39.5, \mathrm{CH}$ & $38.0, \mathrm{CH}$ & $38.2, \mathrm{CH}$ \\
\hline 8 & $49.0, \mathrm{C}$ & $46.7, \mathrm{C}$ & $47.6, \mathrm{C}$ \\
\hline 9 & $208.3, \mathrm{C}$ & 209.6, C & $207.9, \mathrm{C}$ \\
\hline 10 & $210.7, \mathrm{C}$ & $210.5, \mathrm{C}$ & 197.5, C \\
\hline 11 & $41.66, \mathrm{CH}$ & $42.3, \mathrm{CH}$ & 138.6, C \\
\hline 12 & $21.3, \mathrm{CH}_{3}$ & $21.9, \mathrm{CH}_{3}$ & $129.8, \mathrm{CH}$ \\
\hline 13 & $20.7, \mathrm{CH}_{3}$ & $21.6, \mathrm{CH}_{3}$ & $129.8, \mathrm{CH}$ \\
\hline 14 & & & $129.0, \mathrm{CH}$ \\
\hline 15 & & & $133.7, \mathrm{CH}$ \\
\hline 16 & & & $129.0, \mathrm{CH}$ \\
\hline 17 & $23.4, \mathrm{CH}_{2}$ & $23.8, \mathrm{CH}_{2}$ & $23.3, \mathrm{CH}_{2}$ \\
\hline 18 & 122.0, CH & $121.7, \mathrm{CH}$ & $121.8, \mathrm{CH}$ \\
\hline 19 & $131.5, \mathrm{C}$ & $134.5, \mathrm{C}$ & $133.5, \mathrm{C}$ \\
\hline 20 & $26.0, \mathrm{CH}_{3}$ & $25.9, \mathrm{CH}_{3}$ & $25.97, \mathrm{CH}_{3}$ \\
\hline 21 & $18.0, \mathrm{CH}_{3}$ & $18.3, \mathrm{CH}_{3}$ & $18.2, \mathrm{CH}_{3}$ \\
\hline 22 & $30.2, \mathrm{CH}_{2}$ & $30.6, \mathrm{CH}_{2}$ & $30.3, \mathrm{CH}_{2}$ \\
\hline 23 & 121.1, CH & $120.8, \mathrm{CH}$ & $121.0, \mathrm{CH}$ \\
\hline 24 & $134.9, \mathrm{C}$ & $135.0, \mathrm{C}$ & $135.5, \mathrm{C}$ \\
\hline 25 & $26.2, \mathrm{CH}_{3}$ & $26.2, \mathrm{CH}_{3}$ & $26.2, \mathrm{CH}_{3}$ \\
\hline 26 & $18.2, \mathrm{CH}_{3}$ & $18.2, \mathrm{CH}_{3}$ & $18.3, \mathrm{CH}_{3}$ \\
\hline 27 & $28.9, \mathrm{CH}_{2}$ & 27.7, $\mathrm{CH}_{2}$ & $27.9, \mathrm{CH}_{2}$ \\
\hline 28 & $123.4, \mathrm{CH}$ & $123.3, \mathrm{CH}$ & $123.5, \mathrm{CH}$ \\
\hline 29 & 134.5, C & 134.6, C & $134.4, \mathrm{C}$ \\
\hline 30 & $26.1, \mathrm{CH}_{3}$ & $26.1, \mathrm{CH}_{3}$ & $26.00, \mathrm{CH}_{3}$ \\
\hline 31 & $17.9, \mathrm{CH}_{3}$ & $18.0, \mathrm{CH}_{3}$ & 18.0, $\mathrm{CH}_{3}$ \\
\hline 32 & $15.3, \mathrm{CH}_{3}$ & $15.8, \mathrm{CH}_{3}$ & $17.7, \mathrm{CH}_{3}$ \\
\hline 33 & $37.3, \mathrm{CH}_{2}$ & $32.3, \mathrm{CH}_{2}$ & $34.9, \mathrm{CH}_{2}$ \\
\hline 34 & $80.9, \mathrm{CH}$ & $22.8, \mathrm{CH}_{2}$ & 24.6, $\mathrm{CH}_{2}$ \\
\hline 35 & $125.2, \mathrm{CH}$ & $88.3, \mathrm{CH}$ & $88.2, \mathrm{CH}$ \\
\hline 36 & 138.7, C & $73.4, \mathrm{C}$ & $72.3, \mathrm{C}$ \\
\hline 37 & $25.8, \mathrm{CH}_{3}$ & $23.7, \mathrm{CH}_{3}$ & 27.5, $\mathrm{CH}_{3}$ \\
\hline 38 & 18.6, $\mathrm{CH}_{3}$ & $28.2, \mathrm{CH}_{3}$ & 22.7, $\mathrm{CH}_{3}$ \\
\hline
\end{tabular}

${ }^{a}$ Recorded at $150 \mathrm{MHz}$

b Recorded at $100 \mathrm{MHz}$

as evidenced by the ROESY correlations of $\mathrm{H}-6 / \mathrm{H}-23$, $\mathrm{H}-6 \beta / \mathrm{H}-22$, and $\mathrm{H}-6 \alpha / \mathrm{H}-7$. Thus, the structure of 1 was elucidated and named as hypercohone D.

Compound 2 was isolated as colorless oil. Based on the positive HRESIMS $\left(\mathrm{m} / \mathrm{z} 553.3888[\mathrm{M}+\mathrm{H}]^{+}\right.$, calcd for 553.3893), the molecular formula was defined as $\mathrm{C}_{35} \mathrm{H}_{52} \mathrm{O}_{5}$. The IR spectrum showed absorption bands at 3441
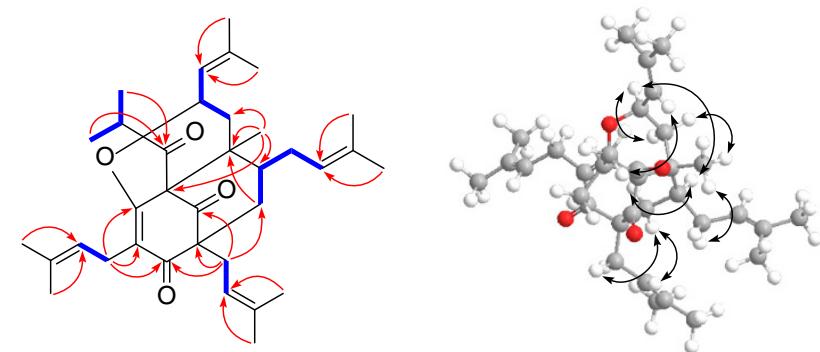

Fig. 1 Key $\operatorname{HMBC}(\longrightarrow),{ }^{1} \mathrm{H}^{-1} \mathrm{H}$ COSY (—), and ROESY $(\longleftrightarrow)$ correlations of $\mathbf{1}$. (Color figure online)

(hydroxyl) and 1724, $1663 \mathrm{~cm}^{-1}$ (carbonyl groups). Extensive analysis of the 1D NMR spectroscopic data (Tables 1 and 2) of $\mathbf{2}$ exhibited a close resemblance with oxepahyperforin (7) [19]. The differences in the 1D spectral data of 2 compared to 7 were that the chemical shifts of C-32 $\left(\delta_{\mathrm{C}} 15.8\right)$, $\mathrm{C}-33\left(\delta_{\mathrm{C}} 32.3\right), \mathrm{C}-34\left(\delta_{\mathrm{C}} 22.8\right), \mathrm{C}-35\left(\delta_{\mathrm{C}} 88.3\right)$, and C-38 $\left(\delta_{\mathrm{C}}\right.$ 28.2) were all little deviated from those of 7 , which indicated that the configuration of C-35 might be differently. Carefully analysis of the ROESY spectrum revealed that $\mathrm{H}-35$ was $\alpha$ oriented, as determined by the correlations of Me-32/H-27, H-7/H-34 $\alpha$, and H-35/H-34 $\alpha$. Therefore, 2 was elucidated to be as the 35-epimer of $\mathbf{7}$ and named hypercohone E (Fig. 2).

Compound 3 possessed a molecular formula of $\mathrm{C}_{38} \mathrm{H}_{50} \mathrm{O}_{5}$, inferred by HRESIMS at $m / z 587.3720[\mathrm{M}+\mathrm{H}]^{+}$(calcd for 587.3736). Its UV absorption exhibited maximum at 278 , 250 , and $205 \mathrm{~nm}$. The IR spectrum displayed peaks at $3440 \mathrm{~cm}^{-1}$ (hydroxyl), $1723 \mathrm{~cm}^{-1}$ (conjugated ketone) and $1653 \mathrm{~cm}^{-1}$ (double-bond). Comparison of the 1D and 2D NMR data indicated that the structures of $\mathbf{3}$ and $\mathbf{7}$ were similar (Tables 1 and 2) [19]. However, the signals for the isopropyl group in $\mathbf{7}$ were replaced by signals for a phenyl group in 3 , which was confirmed by HMBC correlations from both $\mathrm{H}-12\left(\delta_{\mathrm{H}} 7.59, \mathrm{~d}, J=8.2 \mathrm{~Hz}\right)$ and $\mathrm{H}-13\left(\delta_{\mathrm{H}} 7.59, \mathrm{~d}\right.$, $J=8.2 \mathrm{~Hz})$ to $\mathrm{C}-10\left(\delta_{\mathrm{C}} 197.5\right)$ and the proton spin system of $\mathrm{H}-13 / \mathrm{H}-14 / \mathrm{H}-15 / \mathrm{H}-16 / \mathrm{H}-12$ observed from the ${ }^{1} \mathrm{H}-{ }^{1} \mathrm{H}$ COSY spectrum. The ROESY correlations of Me-32/H-27, Me-32/H-33 $\beta, \mathrm{H}-35 / \mathrm{H}-33 \beta$, and $\mathrm{H}-12$ or $\mathrm{H}-13 / \mathrm{H}-35$ deduced that 3 had the same relative configurations as 7 at C-7, C-8 and $\mathrm{C}-35$ and all assigned as $\beta$-orientation. Ultimately, the structure of compound $\mathbf{3}$ was deduced and named as hypercohone $\mathrm{F}$.

Hypercohone G (4) was isolated as a light yellow gum. The molecular formula, $\mathrm{C}_{33} \mathrm{H}_{40} \mathrm{O}_{5}$, was determined by HREIMS ( $/ z / z 516.2871[\mathrm{M}]^{+}$, calcd for 516.2876). The IR spectrum revealed characteristic bands corresponding to the hydroxyl $\left(3451 \mathrm{~cm}^{-1}\right)$, conjugated carbonyl (1715 and $\left.1679 \mathrm{~cm}^{-1}\right)$, and double-bond $\left(1630 \mathrm{~cm}^{-1}\right)$ groups. Detailed comparison of the 1D-NMR data of $\mathbf{4}$ with those of Sampsonol C disclosed that they were closely related and shared a same spiro-cyclic skeleton, except for the 
Table $2{ }^{1} \mathrm{H}$ NMR data for compounds $\mathbf{1}-\mathbf{3}$ in $\mathrm{CD}_{3} \mathrm{OD}(600 \mathrm{MHz}, \delta$ in ppm, $J$ in $\mathrm{Hz}$ )

\begin{tabular}{|c|c|c|c|}
\hline No. & 1 & 2 & 3 \\
\hline \multirow[t]{2}{*}{6} & $\begin{array}{l}\mathrm{H} \propto 1.81, \mathrm{dd}(5.4, \\
13.8)\end{array}$ & $\mathrm{H} \alpha 1.88, \mathrm{~m}$ & $\begin{array}{c}\mathrm{H} \alpha 1.82, \mathrm{dd}(4.9, \\
13.5)\end{array}$ \\
\hline & $\begin{array}{l}\mathrm{H} \beta \text { 1.51, dd (12.6, } \\
\text { 13.8) }\end{array}$ & $\mathrm{H} \beta 1.41, \mathrm{t}(13.2)$ & $\mathrm{H} \beta 1.69, \mathrm{~m}$ \\
\hline 7 & $2.18, \mathrm{~m}$ & $1.91, \mathrm{~m}$ & $2.10, \mathrm{~m}$ \\
\hline 11 & $2.38, \mathrm{~m}$ & $2.33, \mathrm{~m}$ & \\
\hline 12 & $0.97, \mathrm{~d}(6.6)$ & $1.08, \mathrm{~d}(6.6)$ & $7.59, \mathrm{~d}(8.2)$ \\
\hline 13 & $1.03, \mathrm{~d}(6.6)$ & $1.10, \mathrm{~m}$ & $7.59, \mathrm{~d}(8.2)$ \\
\hline 14 & & & $7.31, \mathrm{dd}(7.5,8.2)$ \\
\hline 15 & & & $7.50, \mathrm{t}(7.5)$ \\
\hline 16 & & & $7.31, \mathrm{dd}(7.5,8.2)$ \\
\hline \multirow[t]{2}{*}{17} & 3.15 , dd $(8.4,13.8)$ & $\begin{array}{l}3.18, \text { dd }(7.2, \\
14.4)\end{array}$ & 3.16 , dd $(7.2,13.9)$ \\
\hline & $3.08, \mathrm{dd}(6.6,13.8)$ & $\begin{array}{l}3.12, \mathrm{dd}(6.6, \\
14.4)\end{array}$ & 2.99 , dd $(6.8,13.9)$ \\
\hline 18 & $5.08, \mathrm{~m}$ & $5.09, \mathrm{t}(7.2)$ & $4.90, \mathrm{t}(6.8)$ \\
\hline 20 & $1.68, \mathrm{~m}$ & $1.67, \mathrm{~s}$ & $1.632, \mathrm{~s}$ \\
\hline 21 & $1.68, \mathrm{~s}$ & $1.68, \mathrm{~s}$ & $1.627, \mathrm{~s}$ \\
\hline 22 & $2.41, \mathrm{~m}$ & $\begin{array}{l}2.44, \mathrm{dd}(7.2, \\
13.8) \\
2.37, \mathrm{dd}(7.2 \\
13.8)\end{array}$ & $2.47, \mathrm{~m}$ \\
\hline 23 & $5.06, \mathrm{~m}$ & $4.89, \mathrm{t}(7.8)$ & $5.12, \mathrm{t}(7.1)$ \\
\hline 25 & $1.64, \mathrm{~s}$ & $1.59, \mathrm{~s}$ & $1.67, \mathrm{~s}$ \\
\hline 26 & $1.69, \mathrm{~s}$ & $1.66, \mathrm{~s}$ & $1.67, \mathrm{~s}$ \\
\hline 27 & $\begin{array}{l}\mathrm{H} \alpha 2.05, \mathrm{~m} \\
\mathrm{H} \beta 1.63, \mathrm{~m}\end{array}$ & $\begin{array}{l}\mathrm{H} \alpha 1.94, \mathrm{~m} \\
\mathrm{H} \beta 1.62, \mathrm{~m}\end{array}$ & $\begin{array}{l}\mathrm{H} \alpha 2.16, \mathrm{~m} \\
\mathrm{H} \beta 1.73, \mathrm{~m}\end{array}$ \\
\hline 28 & $5.05, \mathrm{~m}$ & $4.99, \mathrm{t}(7.8)$ & $5.02, \mathrm{t}(7.2)$ \\
\hline 30 & $1.68, \mathrm{~s}$ & $1.68, \mathrm{~s}$ & $1.68, \mathrm{~s}$ \\
\hline 31 & $1.58, \mathrm{~s}$ & $1.57, \mathrm{~s}$ & $1.59, \mathrm{~s}$ \\
\hline 32 & $1.08, \mathrm{~s}$ & $1.03, \mathrm{~s}$ & $1.25, \mathrm{~s}$ \\
\hline \multirow[t]{2}{*}{33} & $\mathrm{H} \alpha 1.70, \mathrm{~m}$ & $\mathrm{H} \alpha 1.65, \mathrm{~m}$ & $\mathrm{H} \alpha 2.06, \mathrm{~m}$ \\
\hline & $\mathrm{H} \beta 2.21, \mathrm{~m}$ & $\mathrm{H} \beta 2.63, \mathrm{t}(14.4)$ & $\mathrm{H} \beta 2.33, \mathrm{~m}$ \\
\hline 34 & 4.97, m & $\begin{array}{l}\mathrm{H} \beta 2.04, \mathrm{~m} \\
\mathrm{H} \alpha 1.77, \mathrm{~m}\end{array}$ & $\begin{array}{l}2.02, \mathrm{~m} \\
1.45, \mathrm{~m}\end{array}$ \\
\hline 35 & $5.18, \mathrm{~d}(8.3)$ & $4.11, \mathrm{~d}(8.4)$ & $3.73, \mathrm{~d}(9.0)$ \\
\hline 37 & $1.76, \mathrm{~s}$ & $1.11, \mathrm{~s}$ & $0.72, \mathrm{~s}$ \\
\hline 38 & $1.69, \mathrm{~s}$ & $1.32, \mathrm{~s}$ & $1.00, \mathrm{~s}$ \\
\hline
\end{tabular}

signal of Me-17 in sampsonol $\mathrm{C}$ was replaced by an isoprenyl group in 4 [21]. This can be revealed by the presence of five carbon signals ascribable for a prenyl at $\delta_{\mathrm{C}}$ 41.4 (t, C-17), 119.8 (d, C-18), 137.0 (s, C-19), 26.3 (q, $\mathrm{C}-20$ ), and 17.8 (q, C-21) (Table 3). The mentioned isoprenyl group was located at C- 6 by the HMBC correlations of $\mathrm{H}-17$ to $\mathrm{C}-1\left(\delta_{\mathrm{C}} 199.3\right), \mathrm{C}-5\left(\delta_{\mathrm{C}} 209.8\right)$, and C-6 $\left(\delta_{\mathrm{C}}\right.$ $65.4)$ coupled with the correlation of $\mathrm{H}-17 / \mathrm{H}-18$ observed in ${ }^{1} \mathrm{H}-{ }^{1} \mathrm{H}$ COSY spectrum. The ROESY interactions between Me-15/H-12, H-12/H-11 $\alpha, \mathrm{H}-11 \beta / \mathrm{H}-8$, and $\mathrm{H}-8 /$
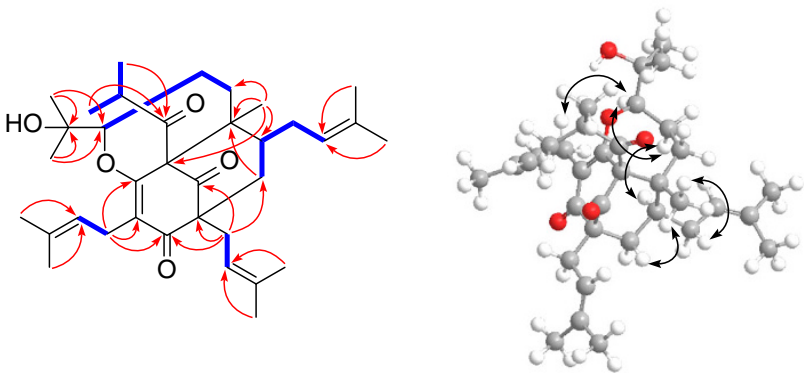

Fig. 2 Key HMBC ( $\longrightarrow$ ), ${ }^{1} \mathrm{H}^{-}{ }^{1} \mathrm{H}$ COSY (—), and ROESY $(\longleftrightarrow)$ correlations of 2 . (Color figure online)
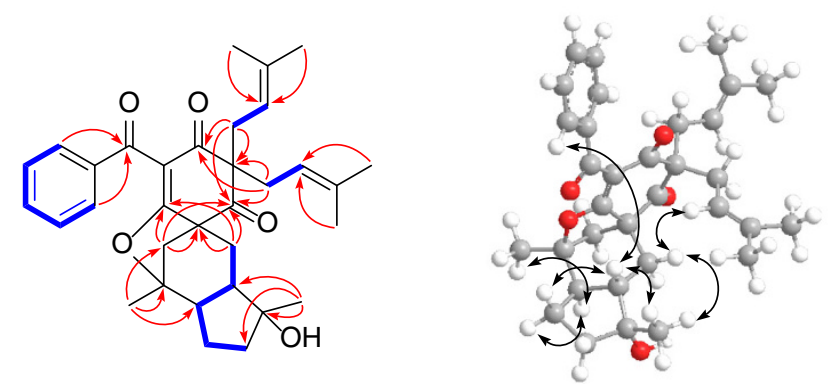

Fig. 3 Key HMBC ( $\longrightarrow$ ), ${ }^{1} \mathrm{H}^{-}{ }^{1} \mathrm{H}$ COSY ( $(\longrightarrow)$, and ROESY $(\longleftrightarrow)$ correlations of 4 . (Color figure online)

Me-16 suggested that 4 had the same relative configurations as Sampsonol C at C-8, C-9, C-12, and C-13 (Fig. 3). Therefore, the structure of $\mathbf{4}$ was established as illustrated and named hypercohone $\mathrm{G}$.

Compounds 1-8 were tested for in vitro inhibitory activities against HL-60, SMMC-7721, A549, MCF-7 and SW480 human tumor cell lines using the MTT method described previously [22]. The results indicated that all the compounds were inactive with $\mathrm{IC}_{50}>30 \mu \mathrm{M}$.

\section{Experiment Section}

\subsection{General Experimental Procedures}

Optical rotations were obtained with a Jasco P-1020 polarimeter. UV spectra were measured on Shimadzu UV2401A spectraphotometer. IR spectra were detected on a Bruker Tensor-27 infrared spectrophotometer with $\mathrm{KBr}$ pellets. 1D and 2D NMR spectra were recorded on Bruker AV-400, and Avance III-600 MHz spectrometers with TMS as the internal standard. Chemical shifts $(\delta)$ were expressed in ppm with reference to the solvent signals. HRESIMS analysis and HREIMS were determined on API QSTAR time-of-flight spectrometer and on Waters Auto spec Premier P776 mass spectrometer. Semi-preparative HPLC was performed on an Agilent 1100 liquid chromatography with a Zorbax SB-C18 $(9.4 \mathrm{~mm} \times 25 \mathrm{~cm})$ 
Table $3{ }^{1} \mathrm{H}$ and ${ }^{13} \mathrm{C}$ NMR data for compound 4 in $\mathrm{CD}_{3} \mathrm{OD}$

\begin{tabular}{|c|c|c|c|c|c|}
\hline Position & $\delta_{\mathrm{C}}^{\mathrm{a}}$ & $\delta_{\mathrm{H}}(J \text { in } \mathrm{Hz})^{\mathrm{b}}$ & Position & $\delta_{\mathrm{C}}^{\mathrm{a}}$ & $\delta_{\mathrm{H}}^{\mathrm{b}}$ \\
\hline 1 & 199.3, C & & 17 & $41.4, \mathrm{CH}_{2}$ & 2.72 , dd $(9.0,13.2)$ \\
\hline 2 & 114.7, C & & & & 2.34 , dd $(7.2,13.2)$ \\
\hline 3 & 179.7, C & & 18 & $119.8, \mathrm{CH}$ & $5.03, \mathrm{~m}$ \\
\hline 4 & $60.1, \mathrm{C}$ & & 19 & $137.0, \mathrm{C}$ & \\
\hline 5 & 209.8, C & & 20 & $26.3, \mathrm{CH}_{3}$ & $1.70, \mathrm{~s}$ \\
\hline 6 & $65.4, \mathrm{C}$ & & 21 & $17.8, \mathrm{CH}_{3}$ & $1.52, \mathrm{~s}$ \\
\hline 7 & 34.1, $\mathrm{CH}_{2}$ & $\begin{array}{l}2.08, \mathrm{~m} \\
1.61, \mathrm{~m}\end{array}$ & 22 & 36.6, $\mathrm{CH}_{2}$ & $2.63, \mathrm{~d}(7.5)$ \\
\hline 8 & $50.8, \mathrm{CH}$ & $1.16, \mathrm{~m}$ & 23 & $121.2, \mathrm{CH}$ & \\
\hline 9 & 77.3, C & & 24 & $136.7, \mathrm{C}$ & $5.04, \mathrm{~m}$ \\
\hline 10 & $40.6, \mathrm{CH}_{2}$ & $1.71, \mathrm{~m}$ & 25 & $26.4, \mathrm{CH}_{3}$ & $1.72, \mathrm{~s}$ \\
\hline 11 & $23.4, \mathrm{CH}_{2}$ & $\begin{array}{l}\mathrm{H} \alpha 1.63, \mathrm{~m} \\
\mathrm{H} \beta 0.99, \mathrm{~m}\end{array}$ & 26 & $18.1, \mathrm{CH}_{3}$ & $1.58, \mathrm{~s}$ \\
\hline 12 & $48.6, \mathrm{CH}$ & $2.04, \mathrm{dt}(6.4,12.0)$ & 27 & 194.1, C & \\
\hline 13 & $93.9, \mathrm{C}$ & & 28 & 139.1, C & \\
\hline 14 & $45.0, \mathrm{CH}_{2}$ & $\begin{array}{l}2.12, \mathrm{~m} \\
1.87, \mathrm{dd}(1.9,11.7)\end{array}$ & $29(33)$ & $130.2, \mathrm{CH}$ & $7.81, \mathrm{~d}(8.3)$ \\
\hline 15 & $21.9, \mathrm{CH}_{3}$ & $1.38, \mathrm{~s}$ & $30(32)$ & $129.7, \mathrm{CH}$ & $7.49, \mathrm{t}(7.5,8.3)$ \\
\hline 16 & 26.6, $\mathrm{CH}_{3}$ & $1.29, \mathrm{~s}$ & 31 & $134.8, \mathrm{CH}$ & $7.63, \mathrm{t}(7.5)$ \\
\hline
\end{tabular}

${ }^{a}$ Recorded at $150 \mathrm{MHz}$

${ }^{\mathrm{b}}$ Recorded at $600 \mathrm{MHz}$

column. Column chromatography was performed on Sephadex LH-20 (GE Healthcare), Silica gel (100-200 and 200-300 mesh, Qingdao Marine Chemical Co., Ltd., Qingdao, China), and Amphichroic RP-18 gel (40-63 $\mu \mathrm{m}$, Merck, Darmstadt, Germany) and MCI gel (75-150 $\mu \mathrm{m}$, Mitsubishi Chemical Corporation, Tokyo, Japan). Fractions were monitored by TLC and spots were visualized by heating silica gel plates sprayed with $10 \% \mathrm{H}_{2} \mathrm{SO}_{4}$ in EtOH.

\subsection{Plant Material}

The aerial parts of $H$. cohaerens $\mathrm{N}$. Robson were collected in Daguan prefecture, Yunnan Province, China, in October 2009. The plant was identified by Dr. En-De Liu, Kunming Institute of Botany, Kunming, China. A voucher specimen was deposited with Kunming Institute of Botany with identification number 200910H01.

\subsection{Extraction and Isolation}

The aerial parts of the air-dried $H$. cohaerens $(10.0 \mathrm{~kg})$ were powdered and percolated with $\mathrm{MeOH}$ at room temperature and filtered. The filtrate was evaporated in vacuo to be concentrated. The crude extract $(1.5 \mathrm{~kg})$ was subjected to silica gel column chromatography eluted with a petroleum ether-acetone gradient $(1: 0,8: 1,4: 1,2: 1$, and $0: 1)$ to produce five fractions, A-E. Fraction B (86.4 g) was separated over a MCI-gel column ( $\mathrm{MeOH}-\mathrm{H}_{2} \mathrm{O}$ from 8:2 to 10:0) to obtain five fractions (Fr. B1-B5). Fr. B2 (22.0 g) was isolated over an MCI gel column ( $\mathrm{MeOH}-\mathrm{H}_{2} \mathrm{O}$ from 85:15 to 100:0) to obtain four fractions (Fr. B2a-B2d). Fr. B2a (5.0 g) was separated on a silica gel column, eluted with petroleum ether-acetone (from 50:1 to 8:2), to yield six fractions (B2a1-B2a6). Fr. B2a2 was purified by repeated silica gel columns and semipreparative HPLC (RP-18, $93 \%$ $\left.\mathrm{CH}_{3} \mathrm{CN}-\mathrm{H}_{2} \mathrm{O}\right)$ to afford 1 (25 mg), 2 (5 mg), 3 (12 mg), oxepahyperforin $(7,14 \mathrm{mg})$. Fr. B3 (13 g) was separated over a MCI-gel column ( $\mathrm{MeOH}-\mathrm{H}_{2} \mathrm{O}$ from 85:15 to 100:0) to obtain five fractions (Fr. B3a-B3e). Fr. B3b was then chromatographed on a silica gel column, eluted with petroleum ether-acetone (from 9:1 to 7:3), to yield seven fractions (Fr. B3b1-B3b7). Subfraction B3b3 (200 mg) was chromatographed by semipreparative HPLC $(90 \% \mathrm{MeOH}-$ $\mathrm{H}_{2} \mathrm{O}$ ) to afford three fractions (Fr. B3b3a-Fr. B3b3c). Fr. B3b3a was separated by a silica gel column, using etherethyl acetate (9:1) as solvent system to obtain uralodin A (5, $16 \mathrm{mg})$ and uralodin B (6, $21 \mathrm{mg})$. Fr. B3b3b and Fr. B3b3c were purified by semipreparative TLC to yield 4 (5 $\mathrm{mg})$ and tomoeone F $(\mathbf{8}, 13 \mathrm{mg})$, respectively. 


\subsection{Hypercohone D (1)}

Light yellow gum; $[\alpha]_{\mathrm{D}}^{24}-97.0(c 0.07, \mathrm{MeOH})$; UV $(\mathrm{MeOH})$ $\lambda_{\max }(\log \varepsilon) 270.0$ (3.69) nm; IR (KBr) $v_{\max } 2963,2927,2856$, 1731, 1665, 1634, 1450, 1380, 1287, 1262, 1121, 1100, 1077, $803 \mathrm{~cm}^{-1}$; ${ }^{1} \mathrm{H}$ and ${ }^{13} \mathrm{C}$ NMR data, see Tables 1 and 2; positive ESIMS $m / z, 557[\mathrm{M}+\mathrm{Na}]^{+}$; positive HRESIMS $\mathrm{m} / \mathrm{z}$ 557.3606 (calcd for $\mathrm{C}_{35} \mathrm{H}_{50} \mathrm{O}_{4} \mathrm{Na}[\mathrm{M}+\mathrm{Na}]^{+}, 557.3606$ ).

\subsection{Hypercohone E (2)}

Colorless oil; $[\alpha]_{\mathrm{D}}^{21}-42.8(c 0.12, \mathrm{MeOH}) ; \mathrm{UV}(\mathrm{MeOH}) \lambda_{\max }$ ( $\log \varepsilon) 260.0(3.82) \mathrm{nm}$; IR (KBr) $v_{\max } 3441,2969,2927$, 2857, 1724, 1663, 1628, 1449, 1382, 1121, $1094 \mathrm{~cm}^{-1} ;{ }^{1} \mathrm{H}$ and ${ }^{13} \mathrm{C}$ NMR data, see Tables 1 and 2; positive ESIMS $\mathrm{m} / \mathrm{z}$ 575 [M+Na $^{+}$; positive HRESIMS $\mathrm{m} / z 553.3888$ (calcd for $\mathrm{C}_{35} \mathrm{H}_{53} \mathrm{O}_{5}[\mathrm{M}+\mathrm{H}]^{+}$, 553.3893).

\subsection{Hypercohone F (3)}

Colorless oil; $[\alpha]_{\mathrm{D}}^{23}-240.8($ c $0.17, \mathrm{MeOH})$; UV $(\mathrm{MeOH})$ $\lambda_{\max }(\log \varepsilon) 278.2$ (4.00), 250.0 (4.13) nm; IR (KBr) $v_{\max }$ 3440, 2967, 2927, 1723, 1693, 1653, 1600, 1448, 1383, 1252, 1217, 1113, $1087 \mathrm{~cm}^{-1} ;{ }^{1} \mathrm{H}$ and ${ }^{13} \mathrm{C}$ NMR data see Tables 1 and 2; positive ESIMS $\mathrm{m} / \mathrm{z} 609[\mathrm{M}+\mathrm{Na}]^{+}$; positive HRESIMS $\mathrm{m} / \mathrm{z} 587.3720$ (calcd for $\mathrm{C}_{38} \mathrm{H}_{51} \mathrm{O}_{5}$ $[\mathrm{M}+\mathrm{H}]^{+}$, 587.3736).

\subsection{Hypercohone G (4)}

Light yellow gum; $[\alpha]_{\mathrm{D}}^{18}-56.1$ ( c $\left.0.16, \mathrm{MeOH}\right)$; UV $(\mathrm{MeOH}) \lambda_{\max }(\log \varepsilon) 253.0(4.32) \mathrm{nm} ; \mathrm{IR}(\mathrm{KBr}) v_{\max } 3451$, 2967, 2931, 2873, 1715, 1679, 1630, 1449, 1382, 1353, $1315,1267,1241,1183,1173,1126,1042,764 \mathrm{~cm}^{-1} ;{ }^{1} \mathrm{H}$ and ${ }^{13} \mathrm{C}$ NMR data see Table 3; positive ESIMS $\mathrm{m} / \mathrm{z} 539$ $[\mathrm{M}+\mathrm{Na}]^{+}$; positive HREIMS $\mathrm{m} / \mathrm{z} 516.2871$ (calcd for $\mathrm{C}_{33} \mathrm{H}_{40} \mathrm{O}_{5}[\mathrm{M}]^{+}$, 516.2876).

\subsection{Cytotoxicity Assays}

The following human tumor cell lines were used: HL-60, SMMC-7721, A-549, MCF-7, and SW-480, which were obtained from ATCC (Manassas, VA, USA). All cells were cultured in RPMI-1640 or DMEM medium (Hyclone, Logan, UT, USA), supplemented with $10 \%$ fetal bovine serum (FBS, Hyclone) at $37{ }^{\circ} \mathrm{C}$ in a humidified atmosphere with $5 \% \mathrm{CO}_{2}$. Cell viability was assessed by conducting colorimetric measurements of the amount of insoluble formazan formed in living cells based on the reduction of 3-(4,5-dimethylthiazol2-yl)-2,5-diphenyltetrazolium bromide (MTT) (Sigma, St. Louis, MO, USA). Briefly, $100 \mu \mathrm{L}$ of adherent cells was seeded into each well of a 96-well cell culture plate and allowed to adhere for $12 \mathrm{~h}$ before test compound addition, while suspended cells were seeded just before test compound addition, both with an initial density of $1 \times 10^{5}$ cells $/ \mathrm{mL}$ in $100 \mu \mathrm{L}$ of medium. Each tumor cell line was exposed to the test compound at various concentrations in triplicate for $48 \mathrm{~h}$, with cis-platin and paclitaxel (Sigma) as positive control. After the incubation, MTT $(100 \mu \mathrm{g})$ was added to each well, and the incubation continued for $4 \mathrm{~h}$ at $37{ }^{\circ} \mathrm{C}$. The cells were lysed with $100 \mu \mathrm{L}$ of $20 \%$ SDS-50 \% DMF after removal of $100 \mu \mathrm{L}$ of medium. The optical density of the lysate was measured at $595 \mathrm{~nm}$ in a 96-well microtiter plate reader (Bio$\mathrm{Rad} 680$ ). The $\mathrm{IC}_{50}$ value of each compound was calculated by Reed and Muench's method [22].

Acknowledgments The work was financially supported by the foundations from NSFC (20972167), the Young Academic Leader Raising Foundation of Yunnan Province (No. 2009CI073), and the foundation from CAS to Dr G. Xu.

Open Access This article is distributed under the terms of the Creative Commons Attribution License which permits any use, distribution, and reproduction in any medium, provided the original author(s) and the source are credited.

\section{References}

1. R. Ciochina, R.B. Grossman, Chem. Rev. 106, 3963-3986 (2006)

2. I.P. Singh, S.B. Bharate, Nat. Prod. Rep. 23, 558-591 (2006)

3. I.P. Singh, J. Sidana, S.B. Bharate, W.J. Foley, Nat. Prod. Rep. 27, 393-416 (2010)

4. L.J. Zhang, C.T. Chiou, J.J. Cheng, H.C. Huang, L.M. Yang Kuo, C.C. Liao, K.F. Bastow, K.H. Lee, Y.H. Kuo, J. Nat. Prod. 73, 557-562 (2010)

5. R.A. Hussain, A.G. Owegby, P. Parimoo, P.G. Waterman, Planta Med. 44, 78-81 (1982)

6. A.L. Piccinelli, O. Cuesta-Robio, M.B. Chica, N. Mahmood, B. Pagano, M. Pavone, V. Barone, L. Rastrelli, Tetrahedron 61, 8206-8211 (2005)

7. U.M. Acuna, M. Figueroa, A. Kavalier, N. Jancovski, M.J. Basile, E.J. Kennelly, J. Nat. Prod. 73, 1775-1779 (2010)

8. T.N. Griffith, L. Varela-Nallar, M.C. Dinamarca, N.C. Inestrosa, Curr. Med. Chem. 17, 391-406 (2010)

9. Z.Y. Xiao, Q. Mu, W.K.P. Shiu, Y.H. Zeng, S. Gibbons, J. Nat. Prod. 70, 1779-1782 (2007)

10. L. Beerhues, Phytochemistry 67, 2201-2207 (2006)

11. N.S. Bystrov, B.K. Chernov, V.N. Dobrynin, M.N. Kolosov, Tetrahedron Lett. 32, 2791-2794 (1975)

12. P. Avato, in Studies in Natural Products Chemistry, vol. 30, ed. by Atta-ur-Rahman (Elsevier, The Netherlands, 2005), pp. $603-634$

13. Y.H. Li, Z.Y. Wu, Flora of China, vol. 50 (Science, Beijing, 1990), p. 12

14. X.W. Yang, X. Deng, X. Liu, C.Y. Wu, X.N. Li, B. Wu, H.R. Luo, Y. Li, H.X. Xu, Q.S. Zhao, G. Xu, Chem. Commun. 48, 5998-6000 (2012)

15. X. Liu, X.W. Yang, C.Q. Chen, C.Y. Wu, J.J. Zhang, J.Z. Ma, H. Wang, Q.S. Zhao, L.X. Yang, G. Xu, Nat. Prod. Bioprospect. 3, 233-237 (2013)

16. X. Liu, X.W. Yang, C.Q. Chen, C.Y. Wu, J.J. Zhang, J.Z. Ma, H. Wang, L.X. Yang, G. Xu, J. Nat. Prod. 76, 1612-1618 (2013) 
17. N. Guo, X.Q. Chen, Q.S. Zhao, Acta Bot. Yunnan. 30, 515-518 (2008)

18. X.Q. Chen, Y. Li, X. Cheng, K. Wang, J. He, Z.H. Pan, M.M. Li, L.Y. Peng, G. Xu, Q.S. Zhao, Chem. Biodivers. 7, 196-204 (2010)

19. L. Verotta, G. Appendino, J. Jakupovic, E. Bombardelli, J. Nat. Prod. 63, 412-415 (2000)

20. W. Hashida, N. Tanaka, Y. Kashiwada, M. Sekiya, Y. Ikeshiro, Phytochemistry 69, 2225-2230 (2008)
21. W.B. Xin, X.H. Man, C.J. Zheng, M. Jia, Y.P. Jiang, X.X. Zhao, G.L. Jin, Z.J. Mao, H.Q. Huang, L.P. Qin, Fitoterapia 83, 1540-1547 (2012)

22. M.C. Alley, D.A. Scudiero, A. Monks, M.L. Hursey, M.J. Czerwinski, D.L. Fine, B.J. Abbott, J.G. Mayo, R.H. Shoemaker, M.R. Boyd, Cancer Res. 48, 589-601 (1988) 